\title{
Composites of Reactive Silica Nanoparticles and Poly(glycidyl methacrylate) with Linear and Crosslinked Chains by in situ Bulk Polymerization
}

\author{
Mustafa M. Demir*, Burcu Altın and Serdar Özçelik \\ Department of Chemistry, İzmir Institute of Technology, Gülbahçe Köyü, Urla 35430, İzmir, Turkey
}

Received 25 November 2009; accepted 2 March 2010

\begin{abstract}
Composites of poly(glycidyl methacrylate) (PGMA) and L-lysine-coated silica nanoparticles with varying contents were prepared by in situ bulk polymerization using benzoyl peroxide (BPO) as free radical initiator. Silica nanoparticles covered by L-lysine molecules were synthesized using emulsion method. Dynamic light scattering measurements confirmed that the particles are highly monodisperse with the diameter of $10 \mathrm{~nm}$ and free of aggregates in the monomer (glycidyl methacrylate, GMA). Upon polymerization of the homogeneous particle/monomer dispersion, aggregates of individual silica nanoparticles are observed by tapping mode atomic force microscope (AFM). Amine and/or carboxylic acid sites on particle surface covalently react with the oxirane groups of the polymer backbone. The aggregation was substantially suppressed by using a difunctional comonomer divinyl benzene (DVB) in polymerization. A three-dimensional polymer network, $\mathrm{P}(\mathrm{GMA}-\mathrm{DVB})$, forms throughout the system. This structure leads to significant progress in particle dispersion, therefore in physical properties of the resulting composite. We demonstrated that the composites prepared by crosslinked chains are thermally more stable and mechanically stiffer than those prepared by linear ones.
\end{abstract}

(c) Koninklijke Brill NV, Leiden, 2010

Keywords

Addition polymerization, crosslinking, in situ polymerization, aggregation, L-lysine

\section{Introduction}

The study of polymer-based composites has seen explosive growth over the last decade and may rightfully be considered as an important branch of the emerging field of nanotechnology [1, 2]. Technological interest in polymers functionalized by inorganic nanoparticles is increasing due to their potential to enhance various value-added properties, such as barrier properties [3], flame resistance [4], optical properties [5, 6], and thermal resistance [7]. Macroscopic performance of poly-

\footnotetext{
* To whom correspondence should be addressed. E-mail: mdemir@iyte.edu.tr
} 
mer/inorganic nanoparticle composites is mainly determined by the size and size distribution of the particles within the polymer matrix, as well as by the nature of the interface between the organic and inorganic components [8]. A high performance, in all applications, is achieved when the entire population of particles is individually dispersed throughout the polymer matrix and there must be good adhesion at the interface of organics and inorganics. However, due to the surface energy mismatch between the components and the consequent poor adhesion between them, inorganic nanoparticles have a strong tendency to aggregate within the polymer matrix. Inorganics $\left(500-2000 \mathrm{~mJ} / \mathrm{m}^{2}\right)$ have two orders of magnitude higher surface energy than polymers $\left(20-50 \mathrm{~mJ} / \mathrm{m}^{2}\right)$ [9].

One of the common approaches to overcome the aggregation problem is to perform in situ bulk polymerization where nanoparticles are homogeneously dispersed into a monomer and polymerization is carried out in the presence of particles [10, 11]. However, our previous experience showed that polymerization induces aggregation of nanoparticles during polymerization due to interparticle depletion attraction, although nanosized particles are individually dispersed into the monomer [11]. We have proposed that the formation of three-dimensional networks by branching and crosslinking the chains in polymerization suppresses the aggregation and reduces the size of particle domains in the polymer matrix.

In this study, we have produced nanosized silica particles where L-lysine molecules have been grafted on the surface. The particles were dispersed into glycidyl methacrylate (GMA) and polymerization was carried out in situ. Amine and/or carboxylic acid sites on the surface of particles are reacted with the oxirane ring of the GMA residue. A difunctional comonomer, divinyl benzene (DVB), is introduced into polymerization as a crosslinker and a network throughout the system is achieved. The effects of this fully crosslinked structure on particle dispersion, thermal resistance, and surface mechanical response at nanometer scale are examined.

Polymer/silica composites are the most commonly reported ones in literature among the numerous inorganic/organic composite materials [12]. Silica particles are mainly used in polymeric composites to improve mechanical properties and to protect optical clarity of neat polymers [13]. Due to the favorable processing conditions and transparency, silica particles have usually been associated with polyacrylates, particularly polymethylmethacrylate (PMMA) [12, 14]. Polyglycidylmethacrylate (PGMA) has previously been shown to possess similar physical properties with PMMA, for example, film forming property, good mechanical properties, transparency, and high chemical resistance $[15,16]$. In fact, from the chemical point of view, PGMA has a significant advantage over PMMA because it has pendant oxirane rings, which can be opened and various functionalities can be introduced [17]. In this work, taking advantage of this opportunity, silica nanoparticles coated with an amino acid (L-lysine, for the first time as surfactant) were produced such that amine/carboxylic acid groups on the surface of particles are reacted with the oxirane group of GMA. Since the vinyl group residue remains available, the 
particle surface becomes reactive in the course of composite preparation via bulk polymerization.

$\mathrm{SiO}_{2}$ (silica) nanoparticles are generally prepared by the Stöber method [18]. This method has been modified many times, as reported in the literature. One of the main drawbacks of this method is the polydispersity of prepared silica nanoparticles in the size below $100 \mathrm{~nm}$ [19]. Microemulsion-based preparations provide a solution to the problem of polydispersity, and produce monodisperse particles with size down to 10-20 $\mathrm{nm}$. However, the particles prepared with microemulsions are covered by surfactants as capping agents, which may deteriorate applications such as catalysis. In contrast, Yokoi et al. recently reported a method to prepare amino acid assisted preparation of highly monodisperse silica nanoparticles within the size range of 12-23 $\mathrm{nm}$. In this method, L-lysine or L-arginine acting as both reducing agent and catalyst is used in the presence of octane as a co-solvent. We further developed the method introduced by Yokoi et al. to prepare silica particles with a size of sub-10 nm [21].

\section{Experimental}

\subsection{Materials}

Glycidyl methacrylate (GMA) stabilized with 100 ppm hydroquinone monoethyl ether and benzoyl peroxide (BPO) as initiator were supplied by Fluka. Tetraethyl orthosilicate (TEOS 98\%, Aldrich), ammonium hydroxide $\left(\mathrm{NH}_{4} \mathrm{OH} 28-30 \%\right.$, Aldrich), absolute ethanol (99.9\%, Fluka), L-lysine (98\%, Fluka), and octane (99\%, Fluka), were used to prepare silica particles. All chemicals were used without any further purification. Ultra pure water (18 M $\Omega$ ) was used throughout the study. Glassware and plastic-ware were cleaned by soaking them in dilute nitric acid $(10 \%)$ and rinsed with distilled water prior to use.

\subsection{Preparation of L-Lysine-Coated Silica Nanoparticles}

The monodisperse silica nanoparticles were prepared following by a method introduced by Yokoi et al. [21]. This method is based on the hydrolysis and condensation reactions of tetraethyl orthosilicate (TEOS) as a silica source in a mixture of octane and water in the presence of the basic amino acid, L-lysine, instead of ammonia as a catalyst. Octane was used as a co-solvent to reduce electrostatic interactions. In a typical synthesis, $0.1 \mathrm{mmol}(0.146 \mathrm{~g})$ of L-lysine was dissolved in the solution containing $7.7 \mathrm{~mol}(139.0 \mathrm{~g})$ of water and $64.0 \mathrm{mmol}(7.3 \mathrm{~g})$ of octane under vigorous stirring at $60^{\circ} \mathrm{C}$ under $\mathrm{N}_{2}$ atmosphere. The $\mathrm{pH}$ of this mixture was 9.2. This solution was stirred overnight at $60^{\circ} \mathrm{C}$ under $\mathrm{N}_{2}$ atmosphere and $50 \mathrm{mmol}(11.4 \mathrm{ml})$ of TEOS was added dropwise with a rate of one drop/s. An oil bath was used to control the temperature of the reactions. The resulting mixture was directly evaporated at $70^{\circ} \mathrm{C}$ to obtain silica nanoparticles in powder. 


\subsection{Nanocomposite Preparation}

L-Lysine-coated silica nanoparticles were dispersed into GMA and the dispersions were subjected to ultrasonication for $15 \mathrm{~min}$. They were kept overnight to wet the particle surface with monomer. After a second set of sonication, BPO, a free radical initiator, was added to the dispersion. The amount of initiator was adjusted to $1 \%$ with respect to the molar concentration of GMA. A Pyrex chemical test tube was used as a reactor. The dispersion was purged by nitrogen to minimize the effect of oxygen prior to polymerization. To obtain nanocomposites, polymerization was carried out in bulk at $50^{\circ} \mathrm{C}$ in the presence of the particles. The reaction took place overnight and was stopped by quenching to room temperature. The content of silica particles in polymer matrices is in the range of $9.0 \%$ and $15.0 \%$ by mass. For preparation of crosslinked matrix, $0.4 \mathrm{ml}$ of divinyl benzene (DVB) with respect to GMA was introduced into particle/monomer dispersions. P(GMA-DVB) refers to crosslinked polymer matrix throughout the manuscript.

\subsection{Characterization}

Fourier Transform Infrared spectroscopy (FTIR) was performed in both transmission mode and diffusive reflectance mode (DRIFT) using a Perkin Elmer FTIR spectrometer (Spectrum 100). The spectra were recorded with a resolution of $4 \mathrm{~cm}^{-1}$ and averaged over 16 scans. The distribution of particles in the PGMA was investigated with a tapping mode atomic force microscopy (AFM) (Digital Instruments, Nanoscope III). Tapping mode analysis was performed by $\mathrm{Si}_{3} \mathrm{~N}_{4}$ tip in air. The tip has an anisotropic shape with a $7 \mathrm{~nm}$ radius. The spring constant of the tip was $159.2 \mathrm{~N} / \mathrm{m}$. Force curves have been collected in random places on the sample. The crack surface of the nanocomposites was imaged by Scanning Electron Microscopy (SEM) (Philips XL-30S FEG). Thermal analysis of the composites was carried out by using thermal gravimetric analysis (TGA) (Perkin Elmer Diomand) under nitrogen atmosphere with a heating rate of $10^{\circ} \mathrm{C} / \mathrm{s}$. The size of the particles was determined by dynamic light scattering (DLS) using a Zetasizer $3000 \mathrm{HS}$. X-ray diffractograms (XRD) were recorded with Phillips X'pert Pro by using $\mathrm{Cu} \mathrm{K} \mathrm{K}_{\alpha}$ radiation source $(\lambda=0.15418 \mathrm{~nm})$.

\section{Results and Discussion}

\subsection{L-Lysine-Coated $\mathrm{SiO}_{2}$ nanoparticles}

The silica nanoparticles were prepared by emulsion method and characterized using DLS, SEM, and XRD analysis. Figure 1(a) shows SEM micrographs of L-lysinecoated $\mathrm{SiO}_{2}$ particles at different magnifications illustrating that the dispersion consisted of individual silica particles of a diameter around $10 \mathrm{~nm}$. A rather uniform distribution of particles was achieved over the whole field of view more than $4 \mu^{2}$. SEM images also exemplify that L-lysine coated silica particles are redispersible, which is an important asset for nanoparticles. Figure 1(b) represents 

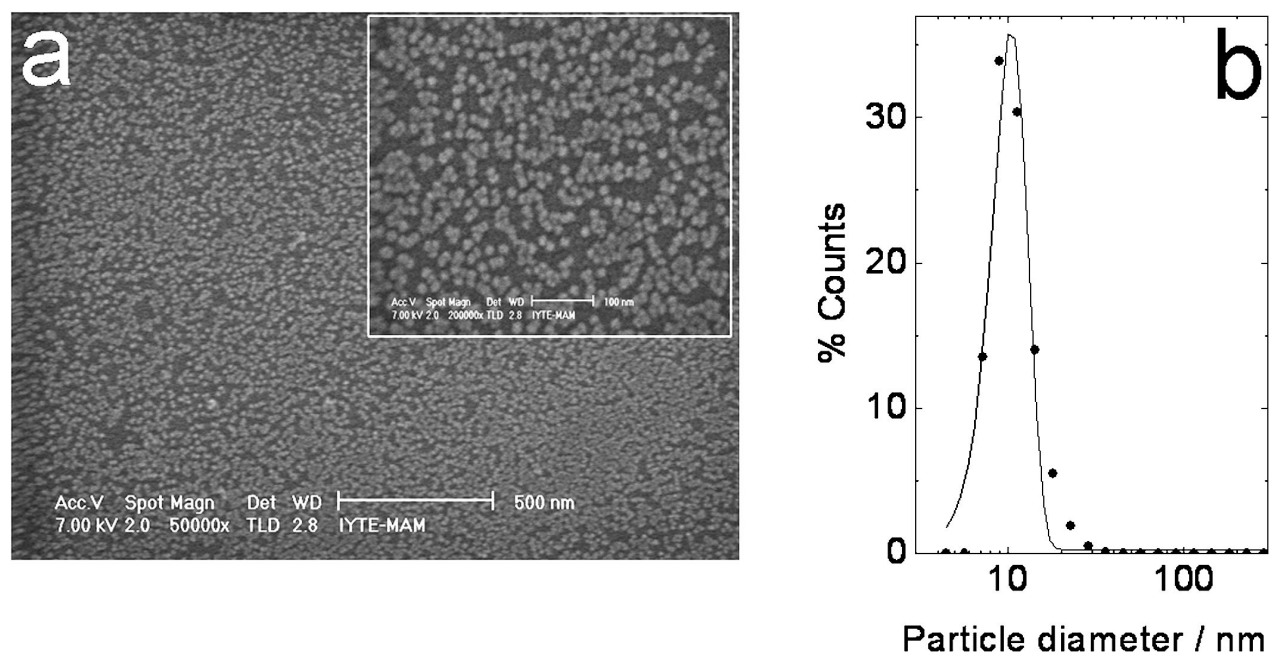

Figure 1. (a) SEM images of L-lysine-coated silica nanoparticles prepared on a substrate after evaporation of solvent at (a) $\times 50000$ and at inset $\times 200000$; (b) DLS number size distributions of silica particles in GMA prior to in situ bulk polymerization.

DLS measurements of L-lysine-coated silica particles in GMA. The particles exhibit monodisperse homogeneous size distribution. The graft density of L-lysine on silica particles was estimated from the mass loss of as synthesized silica particle. The amount of organic in the L-lysine-coated $\mathrm{SiO}_{2}$ particles was $10 \%$ by mass obtained by thermogravimetry. The particles were assumed to have monolayer coverage by L-lysine molecules and the size of the silica particle was estimated from the mean of the number distribution obtained from DLS measurements. The graft density was estimated to be approximately $5 \mu \mathrm{mol} / \mathrm{m}^{2}$.

In order to clarify the interaction between the particle surface and surrounding matrix chains, the silica nanoparticles were treated with GMA at RT for $12 \mathrm{~h}$ and subjected to DRIFT spectroscopy. The spectra of the silica particles, GMA, and GMA-treated silica particles are shown in Fig. 2. The main features showing the grafting of GMA onto L-lysine-coated $\mathrm{SiO}_{2}$ particles are highlighted. The spectrum of L-lysine-coated silica particles is as expected. The silica network is demonstrated by the appearance of symmetric and asymmetric stretching of Si-O-Si at 1080 and $800 \mathrm{~cm}^{-1}$, respectively. The broad band ranging from $3500 \mathrm{~cm}^{-1}$ to $2500 \mathrm{~cm}^{-1}$ is attributed to amine salt and extremely strong hydrogen-bonded amines and surfaceadsorbed water molecules. Primary amines in L-lysine show two broad and weak signals in this region ( $\mathrm{N}-\mathrm{H}$ stretches: $3500-3350 \mathrm{~cm}^{-1}$ and $3350-3200 \mathrm{~cm}^{-1}$ ). GMA has its characteristic signals at $1721 \mathrm{~cm}^{-1}$ from $\mathrm{C}=\mathrm{O}, 1625 \mathrm{~cm}^{-1}$ from $\mathrm{C}=\mathrm{C}$, and 2950, 1170, 907 and $850 \mathrm{~cm}^{-1}$ from oxirane ring. Upon treatment of L-lysine-coated $\mathrm{SiO}_{2}$ particles with GMA, the signals of L-lysine/silica system remain unchanged such as stretching of $\mathrm{Si}-\mathrm{O}-\mathrm{Si}$. However, new signals appear due to grafting of GMA through the oxirane ring. For example, the bands at $1721 \mathrm{~cm}^{-1}$ 
and $1625 \mathrm{~cm}^{-1}$ originating from $\mathrm{C}=\mathrm{O}$ and $\mathrm{C}=\mathrm{C}$ of GMA, respectively, are present in the spectrum of GMA-treated particles. The intensity of these bands is broadened. In addition, the signals originating from the primary amine groups turn into one signal, which is usually observed by secondary amines, upon treatment with GMA indicating that the oxirane group is opened by primary amines. The weak and broad signal at $3500-3350 \mathrm{~cm}^{-1}$ disappears, i.e., the primary amine is replaced with secondary amine through this reaction. This result suggests the successful grafting of GMA to a surface of L-lysine-coated particles.

\subsection{PGMA-Based Nanocomposites}

Polymerization of GMA was carried out in situ using BPO as free radical initiator in the presence of particles as shown in Scheme 1. The reaction continues overnight and the resulting polymer/nanoparticle composite was fractured into two even pieces. The fracture surfaces of PGMA and PGMA-based composites were analyzed by both scanning electron and atomic force microscopes. Figure 3 presents backscattered SEM image of fracture surfaces of PGMA, PGMA/SiO ${ }_{2}$,

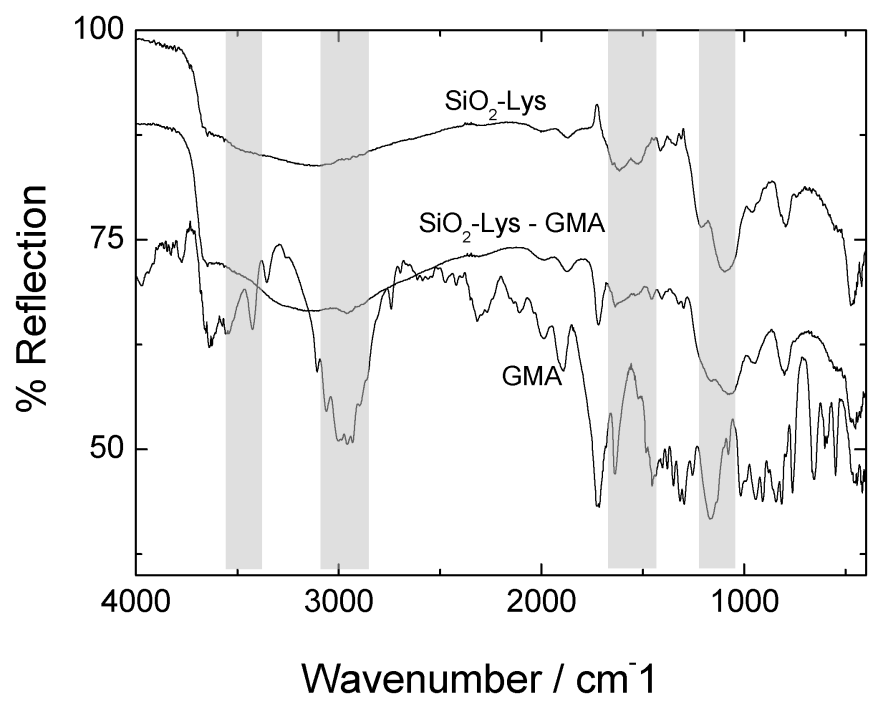

Figure 2. DRIFT spectra of silica nanoparticles and GMA-treated silica particles showing the interaction between amine group on silica particles and oxirane ring of GMA.

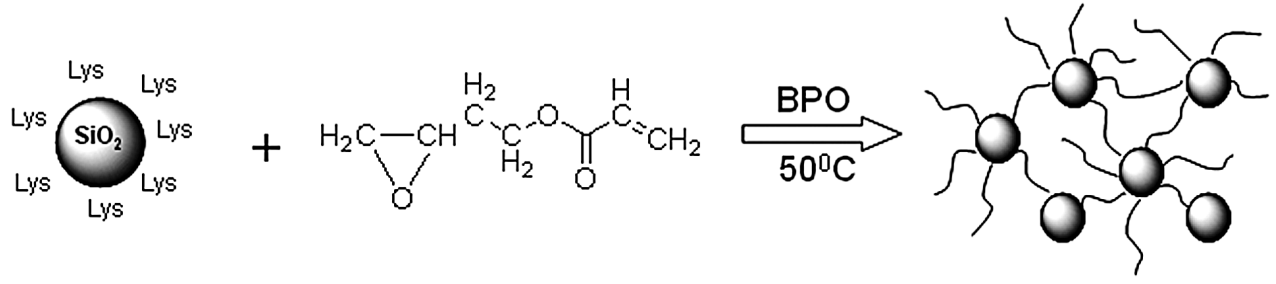

Scheme 1. Schematic view of $\mathrm{PGMA} / \mathrm{SiO}_{2}$ nanocomposite preparation. 
and $\mathrm{P}(\mathrm{GMA}-\mathrm{DVB}) / \mathrm{SiO}_{2}$ when $\mathrm{SiO}_{2}$ content is $10 \%$ by weight. A smooth fracture surface is observed on neat PGMA (Panel a). In contrast, panels b and c illustrate bright features on the images which correspond to aggregates/agglomerates of silica particles. The fracture surface of PGMA/DVB 2 appears to be heterogeneous in nature; however, a more homogeneous surface is observed on the surface of $\mathrm{P}(\mathrm{GMA}-\mathrm{DVB}) / \mathrm{SiO}_{2}$. The smoothness of the surface may indicate relatively brittle fracture and better particle dispersion within crosslinked polymer matrix.

Tapping mode AFM phase imaging with higher magnification was employed to gain insight for particle size and size distribution within polymer matrix. Figure 4 shows AFM phase images of $\mathrm{PGMA} / \mathrm{SiO}_{2}$ and $\mathrm{P}(\mathrm{GMA}-\mathrm{DVB}) / \mathrm{SiO}_{2}$ nanocomposites in panels $a$ and $b$, respectively. Silica particles in nature are harder than the surrounding polymer matrix, so the color of the particles appears lighter than that of the PGMA in the phase images. The size distributions of particle domains obtained from statistical treatment of particles from the AFM images are given in Fig. 5. L-lysine-coated silica particles exhibit individual distribution in GMA with no aggregates (Fig. 1(b)). Both composites contain the same amount of $\mathrm{SiO}_{2}$ nanopar-

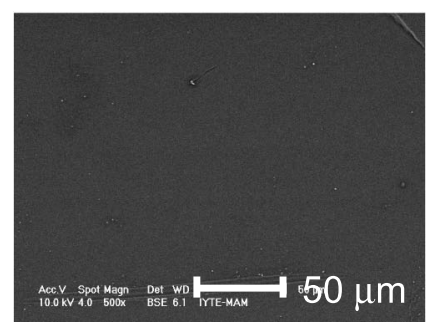

(a)

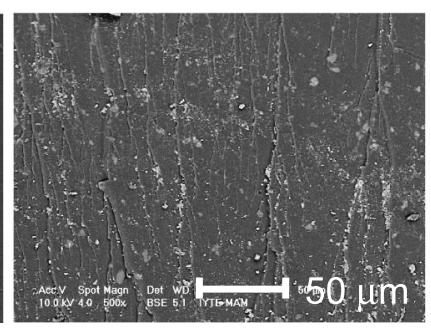

(b)

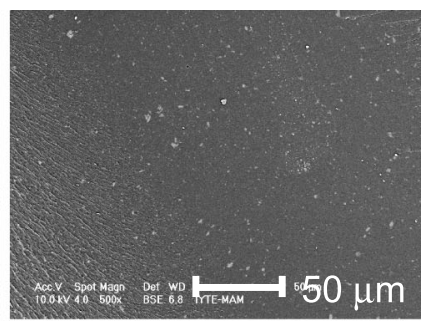

(c)

Figure 3. SEM micrographs showing the fracture surfaces of (a) PGMA, (b) PGMA/SiO 2 and (c) $\mathrm{P}(\mathrm{GMA}-\mathrm{DVB}) / \mathrm{SiO}_{2}$.
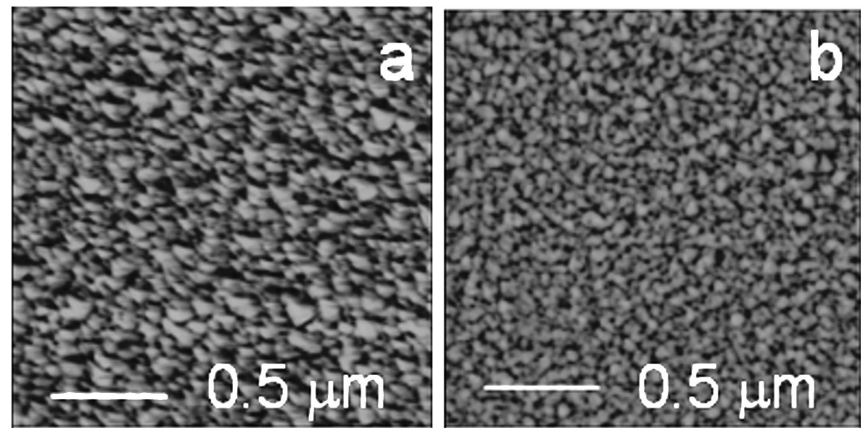

Figure 4. Tapping mode AFM phase images of (a) $\mathrm{PGMA}_{\mathrm{SiO}}$ and (b) $\mathrm{P}(\mathrm{GMA}-\mathrm{DVB}) / \mathrm{SiO}_{2}$ nanocomposites, which contain the same amount of silica nanoparticles (15 wt $\%)$ with identical size and surface modification. Note that the weight fraction is quite high compared with particle size distributions. 
ticles with identical particle size and surface modification. However, particles in crosslinked matrix, $\left.\mathrm{P}(\mathrm{GMA}-\mathrm{DVB}) / \mathrm{SiO}_{2}\right)$, provide smaller domains such that the mean diameter is halved. The reduction of mean diameter by crosslinking may not be satisfactory. However, it has to be noted that the fraction of particles in polymer is $15 \%$ by weight ( $\sim 7 \%$ by volume), which is quite high to reduce the level of aggregation. A similar trend in mean diameter of silica domains upon branching and crosslinking is observed at different fractions given in Table 1. This result can be explained when one considers the mechanism of the entire polymerization process in the presence of particles. In an in situ polymerization, the system is very dynamic. Before the polymerization is initiated, L-lysine-coated silica nanoparticles were freely suspended in the monomer and are able to undergo free Brownian motion. When polymerization is initiated, conversion increases and monomer turns gradually into polymer. In general, the appearance of polymer chains induces interparticle depletion attraction when the formed chains are non-adsorbing to the

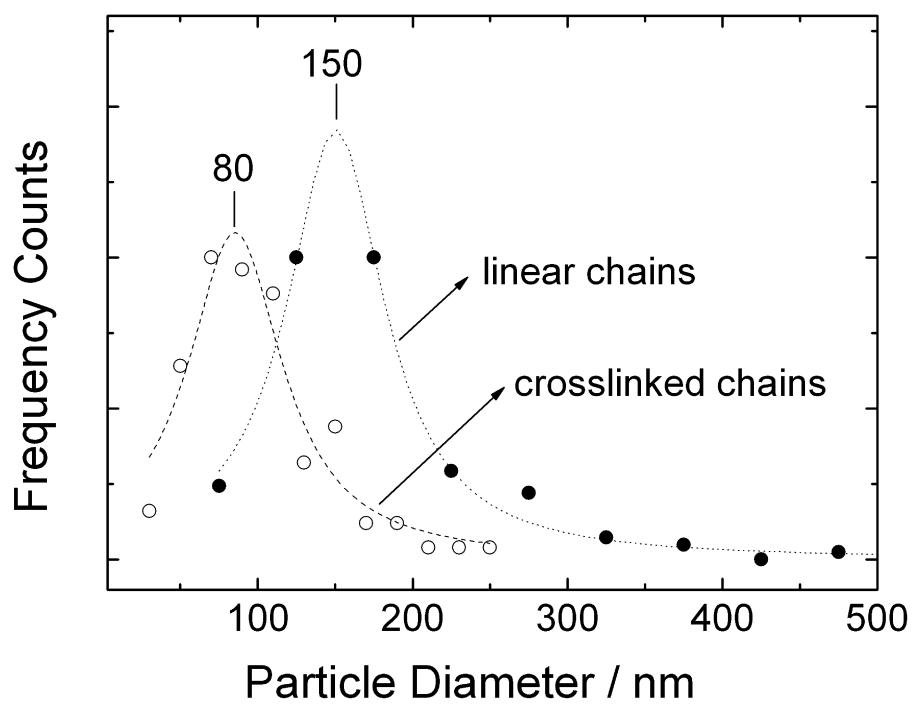

Figure 5. Size distributions of silica particles obtained by DLS in GMA (triangle data points), size distributions of silica domains obtained by statistical treatment of AFM images of PGMA/SiO 2 (open circular data points) and $\mathrm{P}(\mathrm{GMA}-\mathrm{DVB}) / \mathrm{SiO}_{2}$ (close circular data points).

Table 1.

Mean diameter of silica domains $(\mathrm{nm})$ in linear and crosslinked PGMA chains at different silica contents

\begin{tabular}{lll}
\hline Silica \% by mass & PGMA/SiO $_{2}$ & $\mathrm{P}(\mathrm{GMA}-\mathrm{DVB}) / \mathrm{SiO}_{2}$ \\
\hline 15 & 150 & 80 \\
9 & 108 & 70 \\
\hline
\end{tabular}


particle surface. In this particular case, although there is a chemical linkage between the particle surface and the polymer backbone, we also believe that this mechanism takes place particularly at the initial stages of polymerization. As a result, the particles will be pushed against one another and will be separated into large domains of silica particles. We demonstrated that this phase separation process is minimized when a trace amount of a difunctional comonomer, divinyl benzene (DVB), is used in polymerization. The chains are covalently linked to each other through DVB. Eventually, a fully crosslinked polymer network is achieved. This structure exhibits a sudden increase in viscosity of the surrounding matrix via gelation. The particles no longer undergo Brownian motion in a viscous fluid and thereby the diffusion of silica particles is hindered. It is worth pointing out that both composites were insoluble in solvents such as acetone, tetrahydrofurane, and dimethyl formamide, which have been known as good solvents for acrylic polymers. In fact, insolubility of crosslinked matrix, $\mathrm{P}(\mathrm{GMA}-\mathrm{DVB}) / \mathrm{SiO}_{2}$ is not a surprise because of the presence of the covalent linkage between polymer chains. In the case of $\mathrm{PGMA} / \mathrm{SiO}_{2}$, although the polymer chains are linear, it is also insoluble. The existence of a covalent linkage between the particles surface and oxirane group of surrounding GMA residue on polymer backbone imparts this solvent resistance.

The crosslinking process not only enhances dispersibility of the nanoparticles within the polymer matrix but also improves macroscopic properties of the resulting nanocomoposite material. Figure 6 shows degradation profiles of PGMA, $\mathrm{PGMA} / \mathrm{SiO}_{2}$, and $\mathrm{P}(\mathrm{GMA}-\mathrm{DVB}) / \mathrm{SiO}_{2}$ under nitrogen atmosphere from room temperature to $600^{\circ} \mathrm{C}$. Panels a and $\mathrm{b}$ correspond to TGA and DTG, respectively. PGMA as neat polymer is thermally stable till $200^{\circ} \mathrm{C}$. The thermal degradation occurs at $330^{\circ} \mathrm{C}$ and at $410^{\circ} \mathrm{C}$ according to the DTG curves. The overall decomposition process consists of two reactions, namely, depolymerization to monomer, which is the major reaction, and ester decomposition [22]. PGMA produced in the presence of $\mathrm{SiO}_{2}$ (15\% by mass) provides similar degradation profiles with

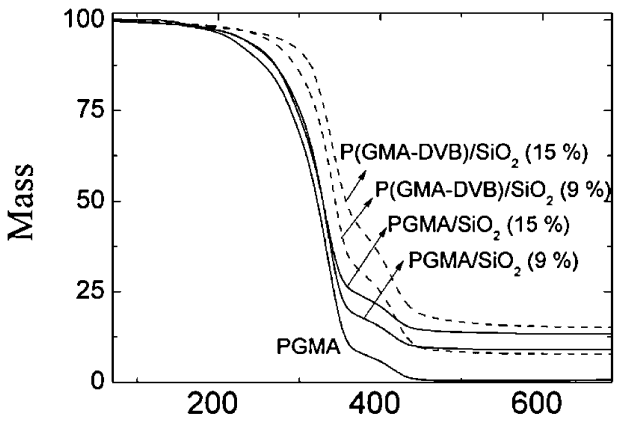

Temperature $\left[{ }^{\circ} \mathrm{C}\right]$

(a)

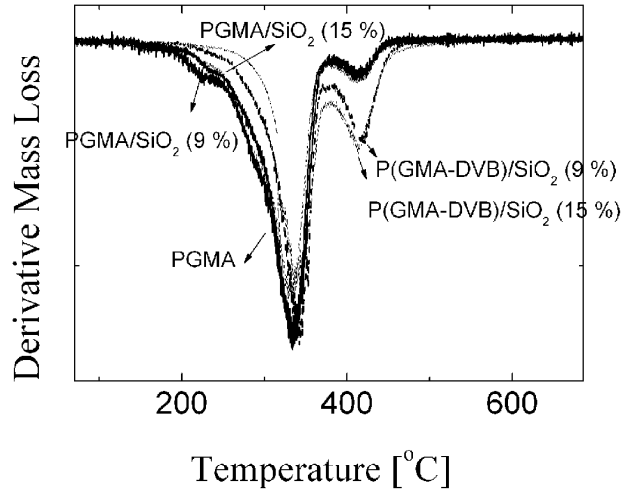

(b)

Figure 6. (a) Mass loss of neat polymer and that of nanocomposites were obtained by thermogravimetry, (b) derivative of mass loss. 
Table 2.

$T_{\mathrm{g}}$ of neat polymer and nanocomposites with linear and crosslinked chains

\begin{tabular}{lr}
\hline Sample & $T_{\mathrm{g}} /{ }^{\circ} \mathrm{C}$ \\
\hline PGMA & 79 \\
PGMA/SiO & 2 \\
P(GMA-DVB)/SiO & 101 \\
\hline
\end{tabular}

slightly higher thermal stability. This result is consistent with literature reports for the PMMA/SiO 2 composite system [4]. However, the thermal stability is remarkably improved by crosslinking of the matrix chains of the composites. In $\mathrm{P}(\mathrm{GMA}-\mathrm{DVB}) / \mathrm{SiO}_{2}$, the onset of degradation is retarded to from $200^{\circ} \mathrm{C}$ to $260^{\circ} \mathrm{C}$. The degradation temperatures were unchanged. However, the area under the derivative curves, i.e., mass loss at the two steps, is different. While the majority of mass loss occurs at the first step in both PGMA and PGMA/SiO 2 , the extent of mass loss is reduced and remarkably shifts to the second of the degradation steps in P(GMA$\mathrm{DVB}) / \mathrm{SiO}_{2}$ (Fig. 6(b)). Thermal stability of the materials can be readily understood when the mass losses are compared at fixed temperature. For instance, the weight loss at $314^{\circ} \mathrm{C}$ is $44 \%$ for neat PGMA, whereas for the composites of PGMA/ $/ \mathrm{SiO}_{2}$ and $\mathrm{P}(\mathrm{GMA}-\mathrm{DVB}) / \mathrm{SiO}_{2}$ containing $15 \mathrm{wt} \% \mathrm{SiO}_{2}$, the weight losses are $36 \%$ and $13 \%$, respectively. As a result, this improvement in thermal properties is originated from the formation of crosslinking between polymer chains, which reduces statistical probability for fragmentation and thus evaporation. In addition, the crosslinking points act as a better barrier for mass transport.

The influence of silica particles on the glass transition behaviour of polymer matrix was studied differential scanning calorimetry (DSC). The values of $T_{\mathrm{g}}$ were taken as a midpoint of the transition and they are presented in Table 2. Neat PGMA has a $T_{\mathrm{g}}$ value of $79^{\circ} \mathrm{C}$, which is consistent with the literature [22]. However, $T_{\mathrm{g}}$ of PGMA/ $/ \mathrm{SiO}_{2}$ was found at $101^{\circ} \mathrm{C}$. $T_{\mathrm{g}}$ of composites strongly depends on the interaction between particles surface and surrounding matrix. For PGMA/SiO ${ }_{2}$ system, the chains are immobilized to the particle surface and this attraction remarkably increases the $T_{\mathrm{g}}$. The crosslinking process links the matrix chains to each other and restricts chain kinetics. This results in a further increase of $T_{\mathrm{g}}$ value to $134^{\circ} \mathrm{C}$. Increase in glass transition confirms the network structure that includes DVB and silica particle surface as crosslinking points.

In order to elucidate the effect of particle dispersion and chemical crosslinking of matrix chains, PGMA and its composites, namely PGMA/SiO ${ }_{2}$ and P(GMA$\mathrm{DVB}) / \mathrm{SiO}_{2}$ at $10 \mathrm{wt} \%$ particles, were analyzed by AFM indentation. In a typical measurement, sharp diamond tip was indented and subsequently imaged by tapping mode imaging. Figure 7 shows an AFM topography image (panel a), AFM phase image (panel b), and cross-section profile (panel c) of the indentation impression 

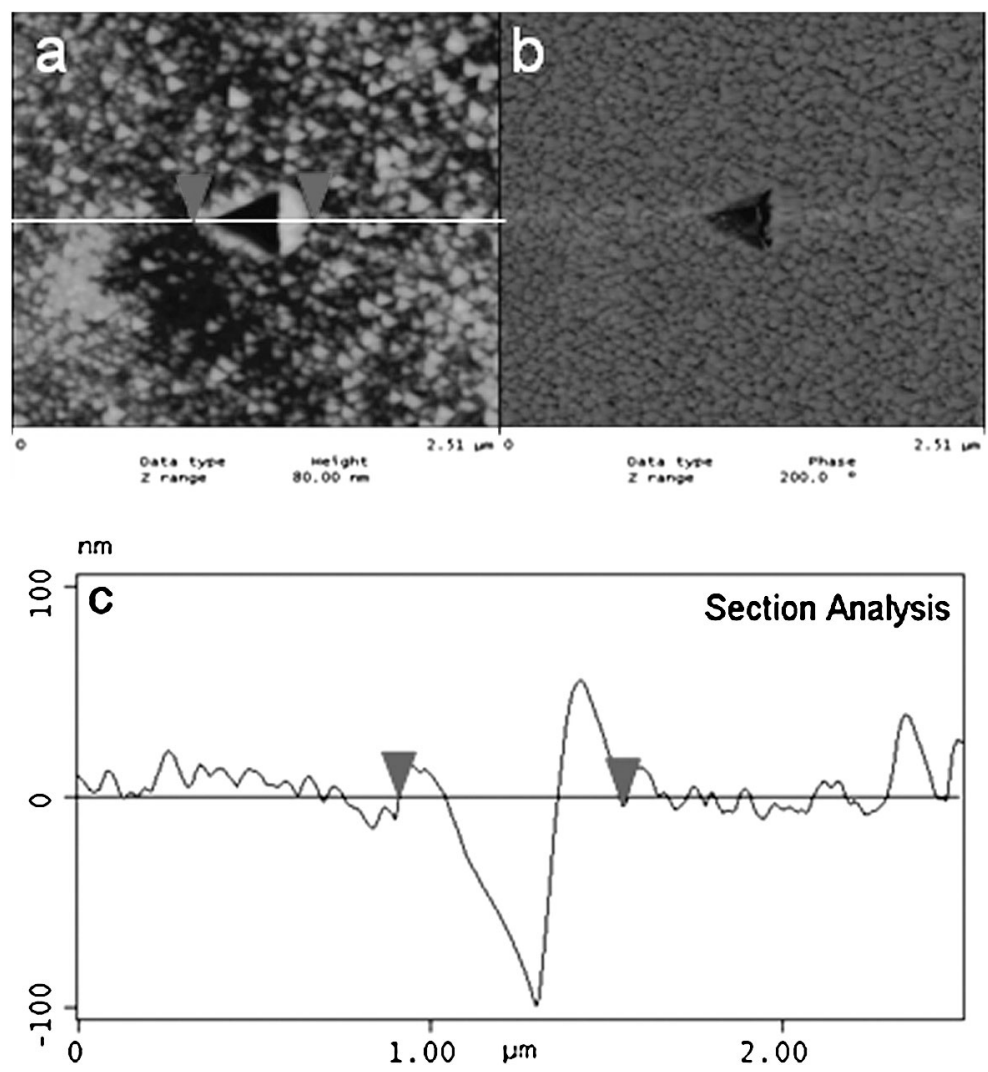

Figure 7. Perspective visualization of AFM data topography (panel a) and phase (panel b) for a nanoindentation in PGMA/SiO $\mathrm{S}_{2}$ at $9 \%$ of silica particles by mass, (c) section analysis of indented region. Note the surface at the edge of the indent is flat as assumed but raised due to 'pile up'.

on PGMA/SiO 2 . Triangular impressions were produced on the PGMA/SiO 2 due to the triangular cross-section of the probe tip. The sample was indented by ploughing (plastic deformation) with pile-up around the indentation tracks with no indentation debris (panel c). Representative load-penetration depth curves of neat PGMA, PGMA/SiO 2 and $\mathrm{P}(\mathrm{GMA}-\mathrm{DVB}) / \mathrm{SiO}_{2}$ are shown in Fig. 8. For all force curves, no discontunity or steps were found on the loading curves, indicating that no cracks formed during indentation. The load-unload curves of the composites have different deformation paths. Material hardness can be inferred from the initial slope of unloading curve through a steeper loading curve and a small residual depth at the point of loading $[23,24]$. In indentation curves, generally, the steeper the slope of the unloading portions of an indentation curve, the stiffer the material. Figure 8 indicates that the linear neat polymer is the least stiff one. As linear PGMA polymers are associated with silica nanoparticles $\left(\mathrm{PGMA} / \mathrm{SiO}_{2}\right)$, shorter residual depth and higher slope are obtained. The presence of silica, which is harder than the surrounding matrix chains increases the hardness of the composite. On the other hand, the 


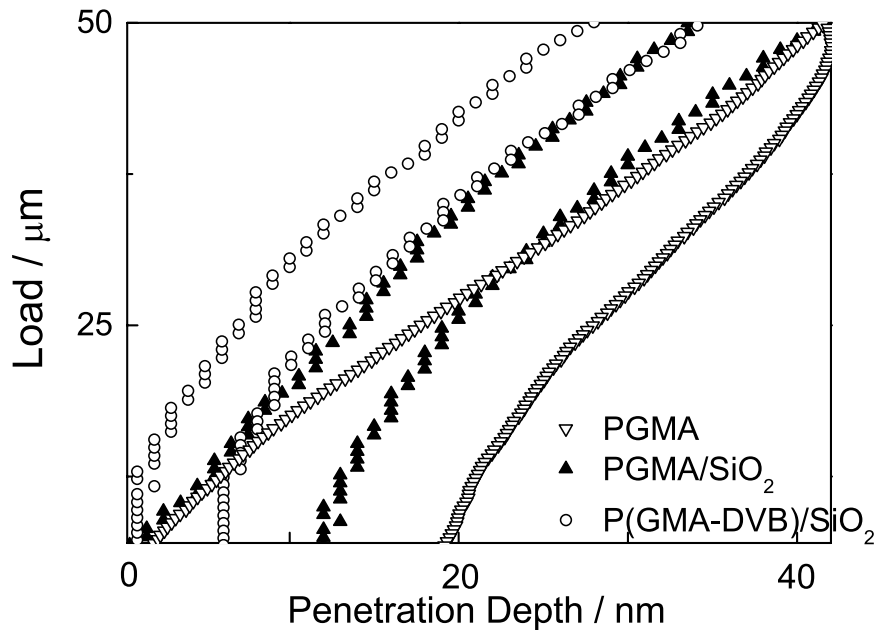

Figure 8. Load-unload curves for PGMA, $\mathrm{PGMA} / \mathrm{SiO}_{2}$ and $\mathrm{P}(\mathrm{GMA}-\mathrm{DVB}) / \mathrm{SiO}_{2}$ nanocomposites when silica content is $10 \%$ by weight.

curve for the composite prepared with crosslinked chains exhibits the further higher slope and shorter residual depth than the one prepared by the linear one. This might be a consequence of better dispersion of the silica particles and less flexibility of the cross-linked chains.

\section{Conclusion}

Composites based on L-lysine-coated silica nanoparticles and PGMA were prepared by in situ bulk polymerization. PGMA chains were covalently linked to the particle surface via opening of the oxirane ring with L-lysine. L-Lysine, an aminoacid, was shown to be a quite efficient surfactant to stabilize silica nanoparticles in an organic medium. We believe that this molecule can be applied for stabilization of other metal oxide colloids. The architecture of in situ formed PGMA chains was found to have a strong influence on the state of silica nanoparticle dispersion in the resulting polymer matrix. The size of silica domains in the crosslinked matrix was remarkably reduced as compared to ones prepared by linear chains. Therefore, homogeneous particle dispersion leads to a significant improvement in both thermal stability and surface stiffness compared to the composite prepared by linear chains.

In summary, in situ polymerization and crosslinking of GMA in the presence of L-lysine-coated silica nanoparticles provide a homogeneous composite that improves thermal and surface mechanical properties.

\section{Acknowledgements}

The authors thank the Reviewer for useful suggestions and members of Materials Research Center of IYTE for microscopy work. M. M. D. acknowledges finan- 
cial support from The Scientific and Technological Research Council of Turkey (TUBITAK) research project encoded with TBAG-109T905. S. O. thanks both State Planning Organization (DPT-2003K120690-6) of Turkish government and TUBITAK (TBAG-108T446) for the financial support.

\section{References}

1. G. Allegra, G. Raos and M. Vacatello, Theories and simulations of polymer-based nanocomposites: from chain statistics to reinforcement, Prog. Polym. Sci. 33, 683-731 (2008).

2. G. Wegner, M. M. Demir, M. Faatz, K. Gorna, R. Munoz-Espi, B. Guillemet and F. Grohn, in: Polymers and Inorganics: A Happy Marriage?, pp. 95-99. IUPAC Intl Sympos. Adv. Polym. Emerging Technol. Oct. 10-13, Busan, South Korea (2006).

3. S. Hess, M. M. Demir, V. Yakutkin, S. Baluschev and G. Wegner, Investigation of oxygen permeation through composites of PMMA and surface-modified $\mathrm{ZnO}$ nanoparticles, Macromol. Rapid Commun. 30, 394-401 (2009).

4. T. Kashiwagi, A. B. Morgan, J. M. Antonucci, M. R. VanLandingham, R. H. Harris, W. H. Awad and J. R. Shields, Thermal and flammability properties of a silica-poly(methylmethacrylate) nanocomposite, J. Appl. Polym. Sci. 89, 2072-2078 (2003).

5. L. L. Beecroft and C. K. Ober, Nanocomposite materials for optical applications, Chem. Mater. $\mathbf{9}$, 1302-1317 (1997).

6. M. M. Demir, K. Koynov, U. Akbey, C. Bubeck, I. Park, I. Lieberwirth and G. Wegner, Optical properties of composites of PMMA and surface-modified zincite nanoparticles, Macromolecules 40, 1089-1100 (2007).

7. M. M. Demir, M. Memesa, P. Castignolles and G. Wegner, PMMA/zinc oxide nanocomposites prepared by in-situ bulk polymerization, Macromol. Rapid Commun. 27, 763-770 (2006).

8. A. C. Balazs, T. Emrick and T. P. Russell, Nanoparticle polymer composites: where two small worlds meet, Science 314, 1107-1110 (2006).

9. W. Caseri, Nanocomposites of polymers and metals or semiconductors: historical background and optical properties, Macromol. Rapid Commun. 21, 705-722 (2000).

10. M. Saric, H. Dietsch and P. Schurtenberger, In situ polymerisation as a route towards transparent nanocomposites: time-resolved light and neutron scattering experiments, Coll. Surf. A-Physicochem. Engng Aspects 291, 110-116 (2006).

11. M. M. Demir, P. Castignolles, U. Akbey and G. Wegner, In-situ bulk polymerization of dilute particle/MMA dispersions, Macromolecules 40, 4190-4198 (2007).

12. H. Zou, S. S. Wu and J. Shen, Polymer/silica nanocomposites: preparation, characterization, properties and applications, Chem. Rev. 108, 3893-3957 (2008).

13. M. M. Demir, Y. Z. Menceloglu and B. Erman, Effect of filler amount on thermoelastic properties of poly(dimethylsiloxane) networks, Polymer 46, 4127-4134 (2005).

14. H. Sugimoto, K. Daimatsu, E. Nakanishi, Y. Ogasawara, T. Yasumura and K. Inomata, Preparation and properties of poly(methylmethacrylate)-silica hybrid materials incorporating reactive silica nanoparticles, Polymer 47, 3754-3759 (2006).

15. K. Naitoh, K. Koseki and T. Yamaoka, An aqueous-base developable photoresist based on light-induced cationic polymerization - resist performance of poly(glycidyl methacrylate-comethacrylic acid), J. Appl. Polym. Sci. 50, 243-250 (1993).

16. A. Hayek, Y. G. Xu, T. Okada, S. Barlow, X. L. Zhu, J. H. Moon, S. R. Marder and S. Yang, Poly(glycidyl methacrylate)s with controlled molecular weights as low-shrinkage resins for 3D multibeam interference lithography, J. Mater. Chem. 18, 3316-3318 (2008). 
17. M. M. Demir, G. Ugur, M. A. Gulgun and Y. Z. Menceloglu, Glycidyl-methacrylate-based electrospun mats and catalytic silver nanoparticles, Macromol. Chem. Phys. 209, 508-515 (2008).

18. W. Stöber, A. Fink and E. Bohn, Controlled growth of monodisperse silica spheres in the micron size range, J. Colloid Interf. Sci. 26, 62-69 (1968).

19. S. M. Yang, S. G. Jang, D. G. Choi, S. Kim and H. K. Yu, Nano-machining by colloidal lithography, Small 2, 458-475 (2006).

20. A. Burcu, Synthesis and Characterization of Monodisperse Silica Based Functional Nanoparticles for Multi-Purpose Applications. İzmir Institute of Technology, İzmir, Turkey (2009).

21. T. Yokoi, Y. Sakamoto, O. Terasaki, Y. Kubota, T. Okubo and T. Tatsumi, Periodic arrangement of silica nanospheres assisted by amino acids, J. Amer. Chem. Soc. 128, 13664-13665 (2006).

22. S. Zulfiqar, M. Zulfiqar, M. Nawaz, I. C. McNeill and J. G. Gorman, Thermal-degradation of poly(glycidyl methacrylate), Polym. Degrad. Stabil. 30, 195-203 (1990).

23. E. Wornyo, K. Gall, F. Z. Yang and W. King, Nanoindentation of shape memory polymer networks, Polymer 48, 3213-3225 (2007).

24. M. R. Vanlandingham, S. H. McKnight, G. R. Palmese, J. R. Elings, X. Huang, T. A. Bogetti, R. F. Eduljee and J. W. Gillespie, Nanoscale indentation of polymer systems using the atomic force microscope, J. Adhesion 64, 31-59 (1997). 